\title{
Metabolomic analysis of the saliva of Japanese patients with oral squamous cell carcinoma
}

\author{
MITSUYOSHI OHSHIMA, KEISUKE SUGAHARA, KIYOHIRO KASAHARA and AKIRA KATAKURA \\ Department of Oral Pathobiological Science and Surgery, Tokyo Dental College, Chiyoda, Tokyo 101-0061, Japan
}

Received November 30, 2016; Accepted February 1, 2017

DOI: $10.3892 / o r .2017 .5561$

\begin{abstract}
The aim of the present study was to characterize the metabolic systems in Japanese patients with oral squamous cell carcinoma (OSCC) using capillary electrophoresis-mass spectrometry (CE-MS) metabolome analysis of saliva samples. A previous study showed variations among ethnicities and tumor sites in the saliva metabolome of patients with OSCC using CE-MS. In the present study, saliva was obtained from 22 Japanese patients with OSCC and from 21 healthy controls who visited the Department of Dentistry, Oral and Maxillofacial Surgery, Tokyo Dental Collage Ichikawa General Hospital, Tokyo, Japan, and all samples were subject to comprehensive quantitative metabolome analysis using CE-MS. A total of 499 metabolites were detected as CE-MS peaks in the saliva tested from the two groups. A total of 25 metabolites were revealed as potential markers to discriminate between patients with OSCC and healthy controls: Choline, $p$-hydroxyphenylacetic acid, and 2-hydroxy-4-methylvaleric acid ( $\mathrm{P}<0.001)$; valine, 3-phenyllactic acid, leucine, hexanoic acid, octanoic acid, terephthalic acid, $\gamma$-butyrobetaine, and 3-(4-hydroxyphenyl)propionic acid $(\mathrm{P}<0.01)$; and isoleucine, tryptophan, 3-phenylpropionic acid, 2-hydroxyvaleric acid, butyric acid, cadaverine, 2-oxoisovaleric acid, N6,N6,N6-trimethyllysine, taurine, glycolic acid, 3-hydroxybutyric acid, heptanoic acid, alanine, and urea $(\mathrm{P}<0.05$, according to the Wilcoxon rank sum test). A previous study by Sugimoto and co-workers detected 24 discriminatory metabolites, 7 of which (taurine, valine, leucine, isoleucine, choline, cadaverine, and tryptophan) were also detected in the present study. In the present study, however, choline, metabolites in the branched chain amino acids (BCAA) cycle, urea, and 3-hydroxybutyric acid were also characterized. Choline and metabolites of the BCAA cycle have previously been reported in OSCC using metabo-
\end{abstract}

Correspondence to: Professor Akira Katakura, Department of Oral Pathobiological Science and Surgery, Tokyo Dental College, 2-9-18 Misaki-cho, Chiyoda-ku, Tokyo 101-0061, Japan

E-mail: katakura@tdc.ac.jp

Key words: biomarker, capillary electrophoresis-mass spectrometry, metabolome, Japanese, oral squamous cell carcinoma, saliva lome analysis. To the best of our knowledge, no previous reports have identified urea and 3-hydroxybuyric acid in the metabolome of patients with OSCC. These findings suggest the usefulness of metabolites as salivary biomarkers for Japanese patients with OSCC. Further studies using larger patient cohorts should be conducted to validate these results.

\section{Introduction}

Oral squamous cell carcinoma (OSCC) is a common malignancy that affects $\sim 300,000$ individuals per year worldwide (1). OSCC is often associated with loss of eating and speech functions, disfigurement, and psychological distress. The primary treatment for OSCC is surgical intervention. Despite considerable advances in the treatment of OSCC over the past two decades, the overall disease outcomes have improved only modestly (2). Local tumor recurrence affects $\sim 60 \%$ of patients, and metastasis develops in $\sim 15-25 \%$ of patients (3). The prevention and management of OSCC will greatly benefit from the identification of molecular markers and targets indicative of the disease $(4,5)$.

Over the course of the last 20 years, saliva has been used to evaluate periodontal disease and the risk of dental caries. It has recently been reported that biomarkers for various diseases, including cancer, may be identified in the saliva, indicating the potential value of saliva as a test sample instead of blood. Recently, salivary diagnosis using various biochemical analytical techniques for the detection of breast and pancreatic cancers has been developed (6).

Using two-dimensional electrophoresis for whole saliva, which can be easily sampled in a non-invasive manner, Katakura et al (7) successfully identified an enolase 1 that is characteristically expressed in the whole saliva of patients with oral cancer. Therefore, the research program of the present authors has continued to focus on salivary metabolomics in our conducting a metabolome analysis and attempting a simultaneous exhaustive search for low-molecular-weight markers for the identification of a plethora of metabolites. Sugimoto et al (6) reported 24 candidate metabolites from saliva samples that were able to serve as biomarkers for cancer patients of various races, geographic regions, and tumor types. This previous study used capillary electrophoresis-mass spectrometry (CE-MS), which is a combined method that has been adapted for the high-resolution separation of ionic compounds, and may be used for metabolome analysis. 
The purpose of the present study was to identify metabolic biomarkers in Japanese patients with OSCC using CE-MS metabolome analysis of saliva.

\section{Materials and methods}

Subjects. Saliva was obtained from Japanese patients with OSCC $(n=22)$ and from healthy controls $(n=21)$ who visited the Department of Dentistry, Oral and Maxillofacial Surgery, Tokyo Dental Collage Ichikawa General Hospital, Tokyo, Japan between September 2013 and March 2015. None of the patients had received any prior treatment in the form of chemotherapy or radiotherapy, and no patient had a history of prior malignancy; information regarding the samples is summarized in Table I. Healthy controls were selected amongst individuals that did not have a history of mucosal diseases in the oral cavity, immunodeficiency, autoimmune disorders, hepatitis, or human immunodeficiency virus (HIV) infection. Written informed consent was obtained from all the subjects. The present study was approved by the ethics committee of Tokyo Dental College (Tokyo, Japan; no. 105).

Sample collection and preparation. All subjects received professional mechanical tooth cleaning by a dental hygienist the day prior to sample collection, and saliva was collected at 8:00 a.m. the following morning under fasting conditions after sufficient gargling and other oral hygiene steps. The subjects were instructed to spit into 50-cc tubes, which were placed in a Styrofoam cup filled with crushed ice. The subjects were reminded not to cough up mucus. It usually took 5-10 min to collect $5 \mathrm{ml}$ of unstimulated saliva. Saliva collection was performed in a restful private room. The saliva samples were centrifuged at 2,600 x g for $15 \mathrm{~min}$ at $4^{\circ} \mathrm{C}$, and spun for a further $20 \mathrm{~min}$ in cases where incomplete separation was observed. After the impurities in the saliva were percolated with a centrifugal filter (Nanosep ${ }^{\circledR}$; Pall Corporation, Port Washington, NY, USA), equal amounts of the supernatant were transferred to two fresh tubes, and the samples were processed and frozen within $30 \mathrm{~min}$. Frozen saliva was thawed and dissolved at room temperature. Prior to the metabolome analyses, each saliva sample (45 $\mu \mathrm{l})$ was added to $5 \mu \mathrm{l}$ Milli-Q water (Merck Millipore, Billerica, MA, USA) containing internal standards and $20 \mathrm{mM}$ each of methionine sulfone, D-camphor-10-sulfonic acid (Wako Pure Chemical Industries, Ltd. Osaka, Japan), 2-(n-morpholino)ethanesulfonic acid (Dojindo Molecular Technologies, Inc., Kumamoto, Japan), 3-aminopyrrolidine (Sigma-Aldrich Japan K.K., Tokyo, Japan), and trimesate (Wako Pure Chemical Industries, Ltd.).

CE-MS metabolome analysis. Cation analysis was performed using a CE capillary electrophoresis system (G1600AX), a G6220A LC/MSD time-of-flight (TOF) system, a 1100-series isocratic high-performance liquid chromatography (HPLC) pump, a CE-MS adapter kit, and a CE-electrospray ionization (ESI)-MS sprayer kit (Agilent Technologies GmbH, Waldbronn, Germany). Anion analysis was performed using a CE capillary electrophoresis system (G1600AX), a G1969A LC/MSD TOF system, a 1100-series isocratic HPLC pump, a CE-MS adapter kit, and a CE-ESI source-MS sprayer kit (Agilent Technologies $\mathrm{GmbH}$ ). For the cation and anion analyses, the CE-MS adapter
Table I. Clinical characteristics of the patients with OSCC and healthy controls.

\begin{tabular}{|c|c|c|}
\hline Characteristics & $\begin{array}{l}\text { OSCC patients } \\
\quad(n=22)\end{array}$ & $\begin{array}{l}\text { Healthy controls } \\
\qquad(\mathrm{n}=21)\end{array}$ \\
\hline Age (yrs.; mean \pm SD) & $68 \pm 13$ & $56 \pm 8$ \\
\hline \multicolumn{3}{|l|}{ Gender } \\
\hline Male & 13 & 8 \\
\hline Female & 9 & 13 \\
\hline \multicolumn{3}{|l|}{ Tumor site } \\
\hline Tongue & 15 & \\
\hline Gingiva & 6 & \\
\hline Oral floor & 1 & \\
\hline \multicolumn{3}{|l|}{$\mathrm{T}$ classification } \\
\hline $\mathrm{T} 1$ & 7 & \\
\hline $\mathrm{T} 2$ & 7 & \\
\hline $\mathrm{T} 3$ & 1 & \\
\hline $\mathrm{T} 4$ & 7 & \\
\hline \multicolumn{3}{|l|}{$\mathrm{N}$ classification } \\
\hline No & 19 & \\
\hline N1 & 2 & \\
\hline $\mathrm{N} 2$ & 1 & \\
\hline $\mathrm{N} 3$ & 0 & \\
\hline \multicolumn{3}{|l|}{ Stage } \\
\hline I & 7 & \\
\hline II & 7 & \\
\hline III & 1 & \\
\hline IV & 7 & \\
\hline
\end{tabular}

OSCC, oral squamous cell carcinoma; SD, standard deviation; TN(M), tumor-lymph-node-(metastasis) classification, confirmed by a histopathological examination within the 12-month follow-up period.

kit included a capillary cassette that facilitates thermostatic control of the capillary. The CE-ESI-MS sprayer kit simplifies coupling of the CE system with the MS system, and is equipped with an electrospray source. For system control and data acquisition, 3D-CE ChemStation software (rev. A.09.03. SR1 and A.10.02) and Agilent MassHunter software were used for CE and TOF-MS (B.04.00 and B.02.00) analyses, respectively. The original Agilent SST316Ti stainless steel ESI needle was replaced with a passivated SST316Ti stainless steel and platinum needle (passivated with $1 \%$ formic acid and a $20 \%$ aqueous solution of isopropanol at $80^{\circ} \mathrm{C}$ for $30 \mathrm{~min}$ ) for anion analysis.

Processing of CE-TOF-MS data and statistical analysis. The metabolite standards, instrumentation, and CE-TOF-MS conditions used in the present study were identical to those previously described (8), with slight modifications in the lock mass system setting. The metabolites were analyzed using a fused silica capillary (50 $\mu \mathrm{m}$ i.d. $\mathrm{x} 80 \mathrm{~cm}$ total length) with a commercial electrophoresis buffer (Solution ID: H3301-1001 for cation analysis and H3302-1021 for anion analysis; Human Metabolome Technologies, Inc., Yamagata, Japan) as the electrolyte. 
Table II. Significance of candidate metabolomes as biomarkers of OSCC.

\begin{tabular}{llcl}
\hline Peak no. & \multicolumn{1}{c}{ Compound name } & Ratio $^{\mathrm{a}}$ & P-value $^{\mathrm{b}}$ \\
\hline 1 & Choline & 2.5 & $6 . \mathrm{E}-05$ \\
2 & p-Hydroxyphenylacetic acid & 2.7 & 0.001 \\
3 & 2-Hydroxy-4-methylvaleric acid & 2.7 & 0.001 \\
4 & Valine & 2.6 & 0.002 \\
5 & 3-Phenyllactic acid & 1.8 & 0.003 \\
6 & Leucine & 2.5 & 0.004 \\
7 & Hexanoic acid & 3.3 & 0.005 \\
8 & Octanoic acid & 1.8 & 0.007 \\
9 & Terephthalic acid & 2.1 & 0.007 \\
10 & -Butyrobetaine & 1.9 & 0.010 \\
11 & 3-(4-Hydroxyphenyl) propionic acid & 3.0 & 0.010 \\
12 & Isoleucine & 2.7 & 0.011 \\
13 & Tryptophan & 1.9 & 0.014 \\
14 & 3-Phenylpropionic acid & 2.9 & 0.016 \\
15 & 2-Hydroxyvaleric acid & 1.0 & 0.017 \\
16 & Butyric acid & 2.6 & 0.019 \\
17 & Cadaverine & 3.4 & 0.026 \\
18 & 2-Oxoisovaleric acid & 1.6 & 0.030 \\
19 & N6,N6,N6-Trimethyllysine & 2.5 & 0.035 \\
20 & Taurine & 1.9 & 0.035 \\
21 & Glycolic acid & 1.1 & 0.036 \\
22 & 3-Hydroxybutyric acid & 1.6 & 0.037 \\
23 & Heptanoic acid & 1.2 & 0.037 \\
24 & Alanine & 1.3 & 0.046 \\
25 & Urea & 0.7 & 0.026 \\
\hline
\end{tabular}

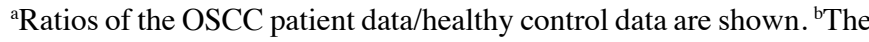
P-values were determined according to the Wilcoxon rank sum test.

Hierarchical cluster analysis was performed using the proprietary software packages, PeakStat and SampleStat, respectively. Detected metabolites were plotted on metabolic pathway maps using Visualization and Analysis of Networks Containing Experimental Data (VANTED) software. Statistical analyses were performed with the Wilcoxon rank sum test to compare the two groups. $\mathrm{P}<0.05$ was considered to indicate a statistically significant value.

Metabolite identification. Although CE-TOF-MS provides accurate molecular mass information at the milli-m/z level, the $\mathrm{m} / \mathrm{z}$ value alone is seldom sufficient to identify a metabolite $(9,10)$. Therefore, in the present analysis a combination of the $\mathrm{m} / \mathrm{z}$ values and the migration times predicted by the artificial neural networks (ANNs) (11) were used to identify the metabolites. In brief, the ANN model was first trained using the measured migration times and molecular descriptors of standard compounds with the net charge calculated from the pKa values. The trained ANN model then predicted the migration times of the candidate metabolites. Herein, the compounds selected as candidates were available in the Kyoto Encyclopedia of Gene and Genomics (KEGG) database (12) and the Human Metabolome Database (HMDB) (13). The composition formulae obtained using the MS data and the matched candidates were confirmed by their isotope distribution patterns.

\section{Results}

Heat map representation of metabolome analysis from patients with OSCC and healthy controls. The saliva samples from 22 patients with OSCC and 21 healthy controls were collected, and metabolites were extracted for CE-MS metabolome analysis.

After having eliminated the extra peaks, such as the isotopic and fragment peaks, the CE-TOF-MS analysis resulted in the detection of 499 peaks in all the saliva samples. Of these, 251 peaks were attributable to known standard metabolites (135 cation peaks and 116 anion peaks). These were identified and quantified with the metabolite standards by matching the $\mathrm{m} / \mathrm{z}$ values with the normalized migration times. The remaining 248 peaks detected belonged to unknown metabolites (45 cation peaks and 203 anion peaks). The score results are presented as a heat map (Fig. 1).

Metabolome pathway in patients with OSCC and healthy controls. A total of 499 metabolites were detected as peaks in patients with OSCC and healthy controls using CE-MS. Of the total number of metabolites, 25 were identified as potential markers that could be used to discriminate between individuals with OSCC and healthy controls (Table II): Choline, p-hydroxyphenylacetic acid, and 2-hydroxy-4-methylvaleric acid $(\mathrm{P}<0.001)$; valine, 3-phenyllactic acid, leucine, hexanoic acid, octanoic acid, terephthalic acid, $\gamma$-butyrobetaine, and 3-(4-hydroxyphenyl)propionic acid $(\mathrm{P}<0.01)$; and isoleucine, tryptophan, 3-phenylpropionic acid, 2-hydroxyvaleric acid, butyric acid, cadaverine, 2-oxoisovaleric acid, N6,N6,N6trimethyllysine, taurine, glycolic acid, 3-hydroxybutyric acid, heptanoic acid, alanine, and urea $(\mathrm{P}<0.05)$. Among these, seven salivary metabolites in patients with OSCC were further characterized: Choline (Fig. 2), metabolites of the branched-chain amino acids (BCAA) cycle (valine, isoleucine, leucine, and 2-oxoisovaleric acid) (Fig. 3), urea (Fig. 4), and 3-hydroxybutyric acid (Fig. 5). Choline showed the greatest statistically significant difference between patients with OSCC and healthy controls in the present study. Sugimoto et al (6) previously reported that choline and the metabolites of the BCAA cycle could be salivary biomarkers for OSCC, but 2-oxoisovaleric acid was not detected in the previous study. Urea was the only metabolite that exhibited a lower level in patients with OSCC compared with healthy controls (Fig. 4). There has been no previous report of 3-hydroxybutyric acid in OSCC (Fig. 5).

\section{Discussion}

Oral cancer, one of the six most common human cancers, is often not diagnosed until it has reached an advanced stage and has a low overall 5-year survival rate of $<50 \%$, which has not essentially changed over the past few decades $(14,15)$. Patients with OSCC often present with symptoms at a late stage, and there is a high recurrence rate following treatment, particularly in patients with neck lymph node metastasis. 


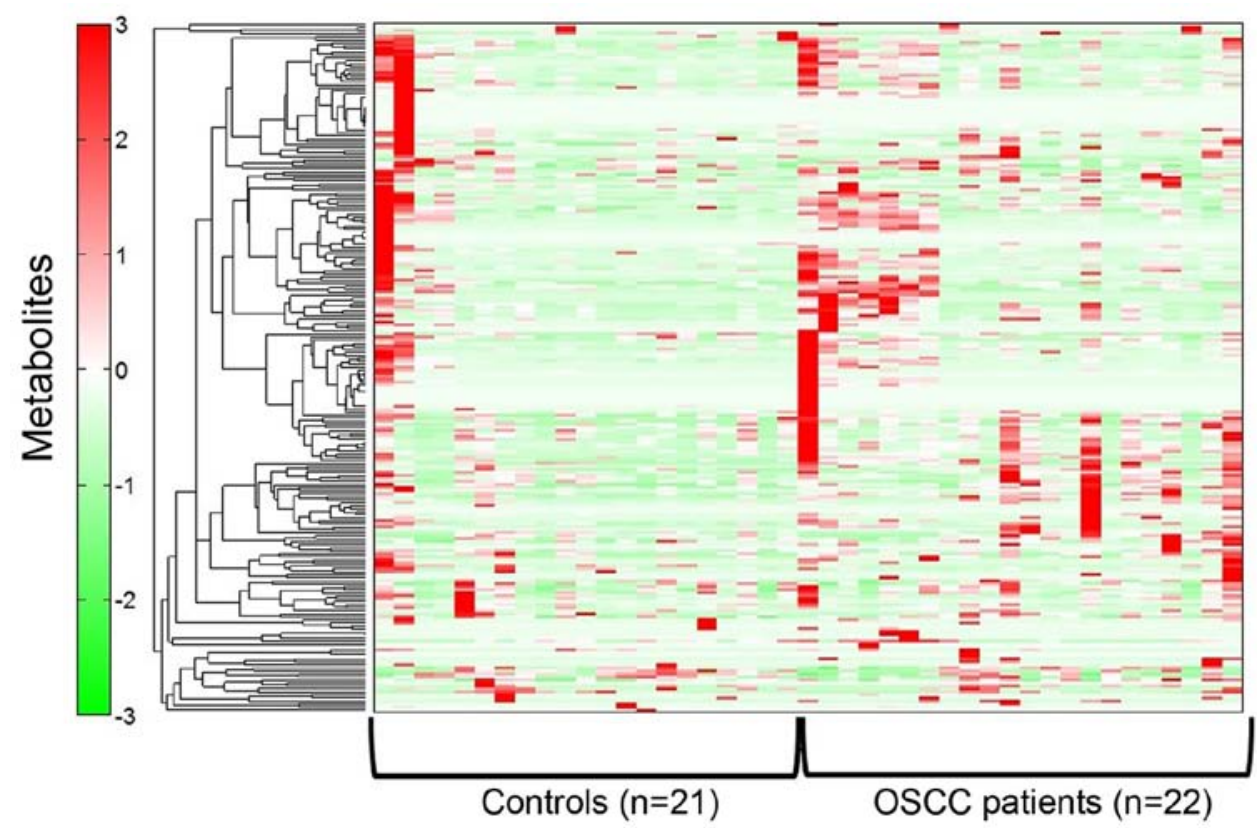

Figure 1. Heat map of 251 peaks, showing significantly different levels ( $\mathrm{P}<0.05$; Steel-Dwass test) between healthy controls ( $\mathrm{n}=21)$ and patients with OSCC $(\mathrm{n}=22)$. Each row shows data for a specific metabolite, and each column represents an individual. The colors correspond to the relative metabolite areas that were converted into Z-scores. OSCC, oral squamous cell carcinoma.

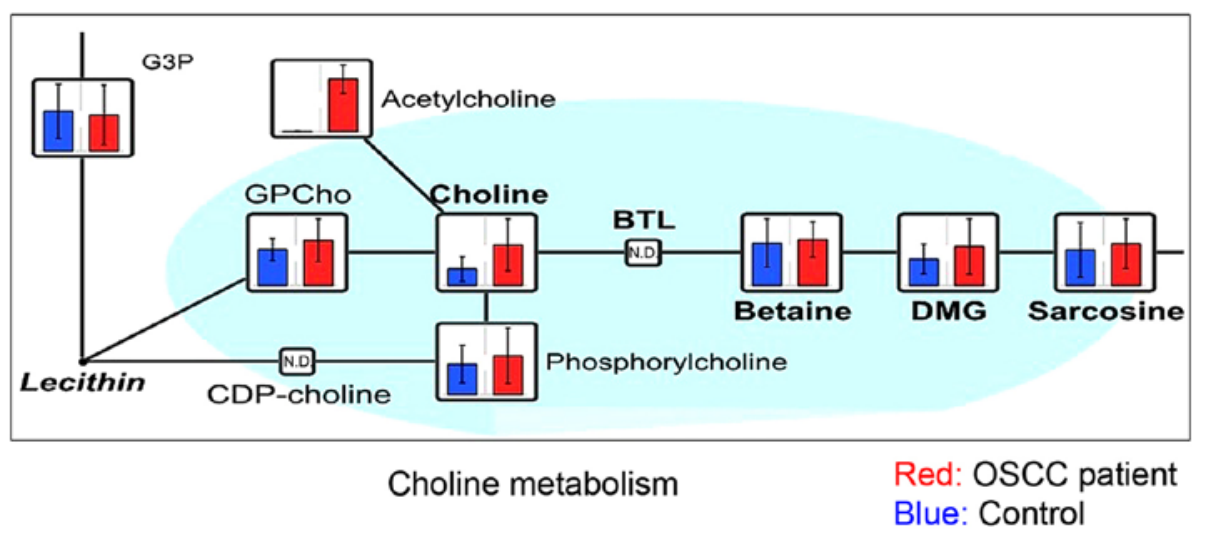

Figure 2. Metabolome data map of the choline pathway. Choline was the most statistically significant metabolite amongst the candidate metabolites detected in the saliva of patients with OSCC. The P-value was 6.E-05. OSCC, oral squamous cell carcinoma; G3P, glycerol 3-phosphate; DMG, dimethylglycine; GPCho, glycerophosphocholine.

The use of saliva as the sample for biomarker detection is a big merit for both the patient and the dentist, because saliva may be collected repeatedly in a non-invasive manner for oral cancer screening. In addition to the straightforward sample collection, other advantages of using a saliva sample compared with other body fluids, such as serum or urine, include the ability to obtain sufficient quantities for analysis and the lower costs of storage and shipping (16). Various changes in a patient's condition are reflected in the blood, but specific markers are observed in the serum for malignant tumors. These changes also have a high possibility of being reflected in the saliva, since serum components are detectable in the saliva (6). In the present study, oral cancer-specific markers with a high discrimination ability were identified and characterized, demonstrating the potential use of salivary metabolomics in OSCC diagnosis. A previous study successfully identified an enolase 1 that is characteristically expressed in the saliva of patients with OSCC using a two-dimensional electrophoretic method (7). Therefore, the focus of the present study was on developing a convenient method for metabolome analysis that may be used to analyze whole saliva samples, which are able to be collected non-invasively and repeatedly. However, it is also important to consider the bacteria present in the oral cavity when using saliva as a biomarker sample. For example, P-hydroxyphenylacetic acid was significantly increased in the OSCC patient saliva compared with healthy control saliva in the present study. P-Hydroxyphenylacetic acid is a metabolic enzyme of tyrosine, which is produced by Porphyromonas gingivalis as a metabolic end-product (17). In the present study, strict criteria for saliva collection were implemented in order to standardize the conditions. All the subjects received professional mechanical tooth cleaning by a dental hygienist 


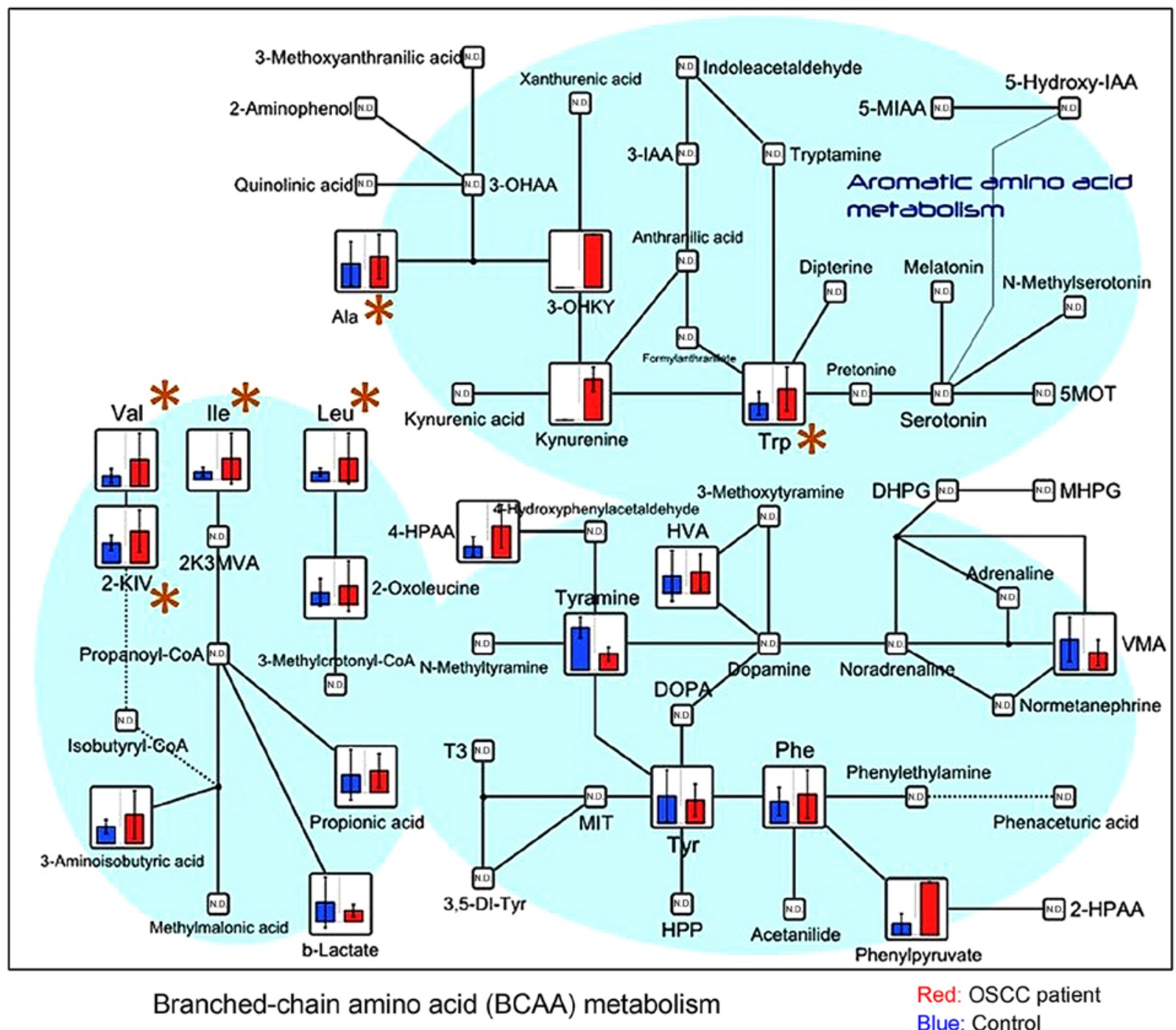

Figure 3. Metabolome data map of metabolites involved in the BCAA and aromatic amino acid pathways, detected in the saliva obtained from patients with OSCC and controls. Levels of valine $(\mathrm{P}=0.002)$, isoleucine $(\mathrm{P}=0.011)$, leucine $(\mathrm{P}=0.004)$, and 2-oxoisovaleric acid $(\mathrm{P}=0.030)$, which are components of the BCAA cycle, exhibited a significant difference between groups. BCAA, branched-chain amino acids; OSCC, oral squamous cell carcinoma; 3-IAA, indole-3-acetic acid; 5-MIAA, 5-methoxyindoleacetic acid; 2-HPAA, 2-hydroxyphosphonocarboxylic acid; MVA, mevalonic acid; KIV, $\alpha$-ketoisovaleric acid; VMA, zanillylmandelic acid; DOPA, dihydroxyphenylalanine; T3, triiodothyronine; MIT, monoiodotyrosine; HPP, hydroxyphenylpyruvate; DHPG, dihydroxyphenylglycine; MHPG, 3-methoxy-4-hydroxyphenylglycol; 5MOT, 5 methoxytryptamine.

the day prior to sample collection, and saliva was collected at 8:00 a.m. the following morning under fasting conditions after sufficient gargling and other oral hygiene steps.

It is widely acknowledged that cancer cells predominantly use glycolysis rather than the oxidative phosphorylation circuit called the tricarboxylic acid (TCA) cycle (Warburg effect) (18). This effect has been detected in stomach cancer and colon cancer tissues based on metabolome analysis with CE-MS (19). Similar metabolic pathway activity has also been reported for oral cancer tissue (6). Since the metabolism observed in the saliva of patients with OSCC is significantly different from the Warburg effect, this suggested that other metabolic pathways should be considered.

Sugimoto et al (6) reported 24 candidate metabolites that were able to serve as biomarkers for OSCC from saliva samples of patients of various races, and 7 of these metabolites (taurine, valine, leucine, isoleucine, choline, cadaverine, and tryptophan) were also detected as potential biomarkers in the present study.
The concentration of choline in the saliva of patients with OSCC was significantly higher compared with that in the saliva of healthy controls; this metabolite exhibited the most significant difference between the two groups. Choline, a quaternary amine, is an essential nutrient that is predominantly supplied through the diet, and choline-containing metabolites are important constituents of the phospholipid metabolism of cell membranes and are associated with malignant transformation, including in breast, brain, and prostate cancer (20). In tumors, choline is highly metabolized to phosphocholine and is oxidized to betaine; hence, a low concentration of choline and high concentrations of phosphocholine and betaine have been observed (21). Furthermore, previous studies have shown that the levels of choline metabolites were higher in tumors compared with benign lesions or normal tissues (22). An excessive increase in plasma choline levels in the tumor cells of patients with breast cancer was also reported (23). Aberrant choline metabolism may be due to enhanced membrane 


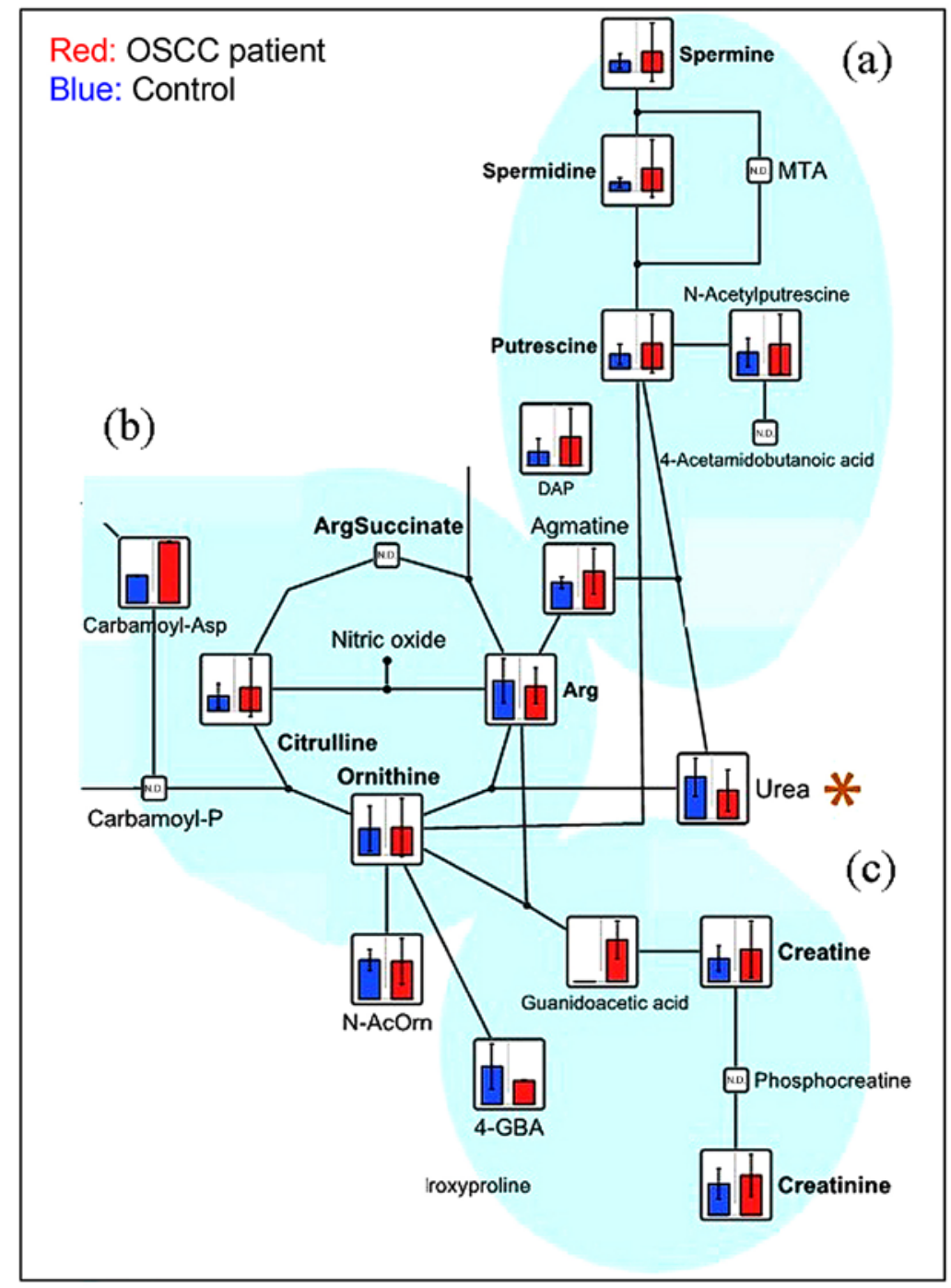

(a) Polyamine metabolism, (b) Urea cycle,

(c) Creatine metabolism

Figure 4. Metabolome data map of metabolites involved in the urea pathway in the saliva obtained from patients with OSCC and healthy controls. Urea showed a significant difference between groups $(\mathrm{P}=0.026)$. OSCC, oral squamous cell carcinoma; MTA, 5'-methylthioadenosine; DAP, 1,3-diaminopropane; 4-GBA, 4-guanidinobutyric acid.

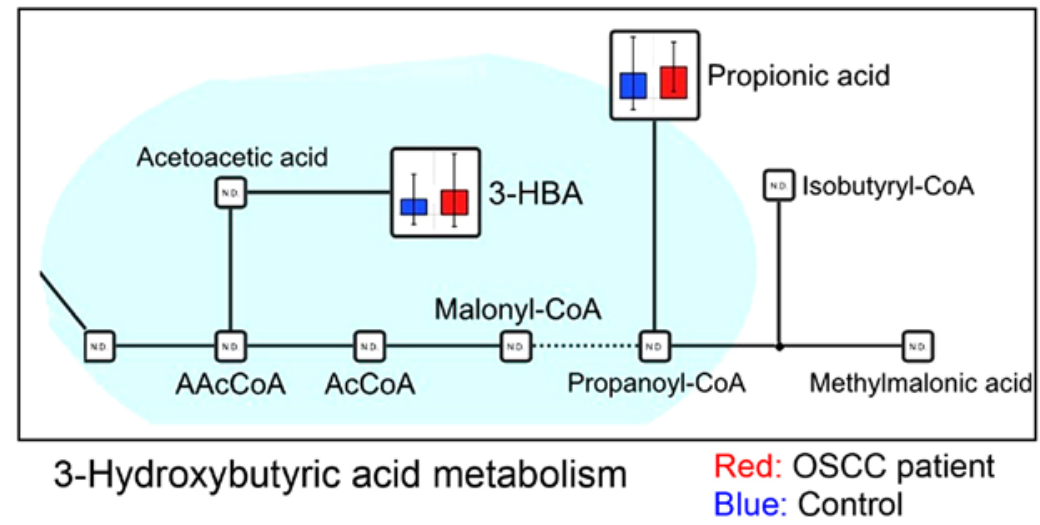

Figure 5. Metabolome data map of the ketone bodies. 3-Hydroxybutyric acid showed a statistically significant difference between groups ( $\mathrm{P}=0.037)$. OSCC, oral squamous cell carcinoma; 3-HBA, 3-hydroxybutyric acid; AAcCoA, acetoacetyl CoA; AcCoA, acetyl CoA.

synthesis and degradation, which reflect the excessive proliferation of cancer cells. The saliva of patients with oral cancer displayed a profile showing increased levels of phosphocholine and glycerophosphocholine (6). In the present study, glycero- 
phosphocholine was detected in certain of the samples, but no statistically significant difference was observed between the two groups. However, this result should be interpreted with caution, since choline is included in various foods. This might not have been an issue in the present study, however, given that the saliva of all the subjects was collected in the morning under fasting conditions after sufficient gargling and oral hygiene. However, to clarify this effect, choline should be detected in paired OSCC and normal tissues simultaneously using CE-MS and another analysis method, such as real-time polymerase chain reaction (RT-PCR) or immunohistochemistry.

BCAAs, such as leucine, isoleucine, and valine, are implicated in various diseases. Branched-chain aminotransferase, which produces a branched chain $\alpha$-keto acid, is an enzyme that catalyzes a reversible amino group transfer reaction in the BCAA degradation system; branched-chain $\alpha$-keto acid dehydrogenase is an enzyme that catalyzes the second step. Finally, acetyl-CoA is formed from leucine, succinyl-CoA and acetoacetate are formed from isoleucine, and succinyl-CoA is formed from valine. The metabolic pathway produces numerous intermediates to be consumed in the TCA cycle. For example, maple syrup urine disease is characterized by dysfunction in BCAA metabolism (24). Valine has been reported as a metabolite that differs significantly in the saliva of patients with uterine (25), colon (26), renal (27), and oral (6) cancer. Leucine levels have been shown to be markedly elevated in women with rectal cancer (28). Cancer cells require excess nutrients and energy to adapt to increased biosynthetic activity, which is correlated with glutamine activity. Several anticancer agents (e.g., l-asparaginase) used in clinical practice utilize mechanisms that inhibit glutamine. Glutamine contributes to the cellular import of leucine, which controls the amino acid/Rag/mammalian target of rapamycin complex 1 (mTORC1) signaling pathway (29). $\alpha$-Keto-carboxylic acid, consisting of amino groups, receives isoleucine from glutamic acid. A significant difference in isoleucine was detected in serum samples of patients with uterine cancer when analyzed by nuclear magnetic resonance (NMR) spectroscopy (30). The levels of isoleucine were also reported to differ markedly in the serum of patients with lung cancer (31), and in patients with schizophrenic disease (32). It was reported that the levels of BCAAs, including leucine, isoleucine, and valine, were significantly higher in cancer patients compared with control subjects (6), and the same results were identified with the patients with OSCC in the present study. The metabolic pathway produces numerous intermediates to be consumed in the TCA cycle. Therefore, metabolism of BCAAs may also serve an important role in the energy production of oral cancer. In the present study, the levels of valine, isoleucine, leucine, and 2-oxoisovaleric acid, which are all involved in the BCAA cycle, significantly differed between the two groups. However, relatively little is known concerning the function of 2-oxoisovaleric acid in general, and it has not been reported in any previous cancer metabolomic study, including those for OSCC.

Urea is a highly soluble organic compound formed in the liver from ammonia produced by the deamination of amino acids. It is the principal end-product of protein catabolism, and accounts for approximately half of the total urinary solids. Urea is formed in a cyclic pathway known as the urea cycle. Urea was shown to be significantly increased in the urine samples of patients with gastric cancer compared with healthy controls (33); however, there has been no previous report of variations in the urea level in patients with OSCC. In the present study, urea was the only metabolite that was significantly higher in the healthy controls compared with patients with OSCC. Urea production is impaired under conditions of poor nutrition and Helicobacter pylori infection. As dietary intake becomes difficult for patients with OSCC due to pain, their protein intake is likely to become insufficient. $H$. pylori produces a urease (34) that catalyzes the conversion of urea into ammonia and carbon dioxide contained in the stomach mucus. Urea produced under the influence of H.pylori metabolism is used in the production of ammonia. No statistically significant differences in urea levels were identified between patients with tongue and gingiva cancer (unpublished data). To the best of our knowledge, no previous metabolomic study of OSCC tumor and saliva samples has identified urea as a marker for OSCC. However, the present findings indicate that decreased urea in the saliva is possibly a biomarker for OSCC. Nevertheless, as mentioned above, there is a requirement for further evaluation of the association between this finding and the metabolism of oral bacteria.

3-Hydroxybutyric acid has an asymmetric carbon atom, and is one of the ketone bodies. It is synthesized in the liver from acetyl-CoA, when the blood glucose concentration, which is used as an energy source, is low. Regarding the increased level of 3-hydroxybutyric acid in the saliva of patients with OSCC, the higher lipid levels in the OSCC saliva may be associated with a higher metabolic turnover and the demand for membrane biosynthesis for cell proliferation, leading to a higher utilization rate of lipids. 3-Hydroxybutyric acid levels are increased in ketosis. 3-Hydroxyisovalerate, which is derived from isovaleryl-CoA, a catabolic intermediate of leucine, has been attracting attention recently as a potential biomarker of ovarian (35), liver (36), pancreatic (37), and gastric (38) cancer, with markedly increased differences observed in the serum or urine. As oral cancer cells use ketone bodies to generate energy, it could possibly be detected in the saliva. Although there is no report of this metabolite in OSCC, 3-hydroxybutyric acid levels in tongue cancer were higher than those in gingiva cancer (unpublished data). In a further study, we will investigate a role and function of 3-hydroxybutyrate in OSCC patient.

In summary, the present analysis of the saliva of Japanese patients with OSCC revealed similar choline and BCAA cycle levels, as identified in the study by Sugimoto et al (6). However, the levels of 3-hydroxybutyric acid and 2-oxoisovaleric acid were higher in the saliva of patients with OSCC compared with the saliva of healthy control subjects, whereas urea levels were lower in Japanese OSCC samples compared with those of healthy controls. The findings in the present study regarding metabolism specific to OSCC may provide a novel strategy for the detection of OSCC, and thereby improve the treatment efficacy. However, in future studies, CE-MS should be combined with other analytical techniques, such as HPLC, RT-PCR and immunochemical staining, and other metabolome analytical techniques, such as NMR and LC-MS spectroscopy. Investigating the association between metabolites and the gene copy number, and gene and protein expression levels in the metabolic pathway of samples from patients with OSCC, may help to elucidate the mechanisms underlying carcinogenesis. 


\section{Acknowledgements}

We thank Professor Nobuo Takano (Oral Cancer Center, Tokyo Dental College), Dr Masahiro Sugimoto (Keio University, Tokyo, Japan), and Dr Kenjiro Kami (Human Metabolome Technologies Inc., Yamagata, Japan) for their technical advice.

\section{References}

1. Sudbø J: Novel management of oral cancer: A paradigm of predictive oncology. Clin Med Res 2: 233-242, 2004.

2. Eheman C, Henley SJ, Ballard-Barbash R, Jacobs EJ, Schymura MJ, Noone AM, Pan L, Anderson RN, Fulton JE, Kohler BA, et al: Annual Report to the Nation on the status of cancer, 1975-2008, featuring cancers associated with excess weight and lack of sufficient physical activity. Cancer 118: 2338-2366, 2012.

3. Genden EM, Ferlito A, Bradley PJ, Rinaldo A and Scully C: Neck disease and distant metastases. Oral Oncol 39: 207-212, 2003.

4. Sabichi AL, Demierre MF, Hawk ET, Lerman CE and Lippman SM: Frontiers in cancer prevention research. Cancer Res 63: 5649-5655, 2003.

5. Spafford MF, Koch WM, Reed AL, Califano JA, Xu LH, Eisenberger CF, Yip L, Leong PL, Wu L, Liu SX, et al: Detection of head and neck squamous cell carcinoma among exfoliated oral mucosal cells by microsatellite analysis. Clin Cancer Res 7: 607-612, 2001

6. Sugimoto M, Wong DT, Hirayama A, Soga T and Tomita M: Capillary electrophoresis mass spectrometry-based saliva metabolomics identified oral, breast and pancreatic cancerspecific profiles. Metabolomics 6: 78-95, 2010.

7. Katakura A, Yamamoto N, Sakuma T, Sugahara K, Onda T, Noguchi S and Shibahara T: A screening test for oral cancer using saliva samples: Proteomic analysis of biomarkers in whole saliva. J Oral Maxillofac Surg 27: 1-5, 2015.

8. Soga T, Baran R, Suematsu M, Ueno Y, Ikeda S, Sakurakawa T, Kakazu Y, Ishikawa T, Robert M, Nishioka T, et al: Differential metabolomics reveals ophthalmic acid as an oxidative stress biomarker indicating hepatic glutathione consumption. J Biol Chem 281: 16768-16776, 2006.

9. Kind $\mathrm{T}$ and Fiehn O: Metabolomic database annotations via query of elemental compositions: Mass accuracy is insufficient even at less than $1 \mathrm{ppm}$. BMC Bioinformatics 7: 234, 2006.

10. Kind $\mathrm{T}$ and Fiehn O: Seven Golden Rules for heuristic filtering of molecular formulas obtained by accurate mass spectrometry. BMC Bioinformatics 8: 105, 2007.

11. Sugimoto M, Kikuchi S, Arita M, Soga T, Nishioka T and Tomita M: Large-scale prediction of cationic metabolite identity and migration time in capillary electrophoresis mass spectrometry using artificial neural networks. Anal Chem 77: 78-84, 2005.

12. Goto S, Okuno Y, Hattori M, Nishioka T and Kanehisa M: LIGAND: Database of chemical compounds and reactions in biological pathways. Nucleic Acids Res 30: 402-404, 2002.

13. Wishart DS, Tzur D, Knox C, Eisner R, Guo AC, Young N, Cheng D, Jewell K, Arndt D, Sawhney S, et al: HMDB: The human metabolome database. Nucleic Acids Res 35: D521-D526, 2007.

14. Vokes EE, Weichselbaum RR, Lippman SM and Hong WK Head and neck cancer. N Engl J Med 328: 184-194, 1993.

15. Banks RE, Dunn MJ,Hochstrasser DF, Sanchez JC, Blackstock W, Pappin DJ and Selby PJ: Proteomics: New perspectives, new biomedical opportunities. Lancet 356: 1749-1756, 2000.

16. Li Y, St John MA, Zhou X, Kim Y, Sinha U, Jordan RC, Eisele D, Abemayor E, Elashoff D, Park NH, et al: Salivary transcriptome diagnostics for oral cancer detection. Clin Cancer Res 10: 8442-8450, 2004.

17. Takahama U, Imamura $H$ and Hirota $S$ : Nitration of the salivary component 4-hydroxyphenylacetic acid in the human oral cavity: Enhancement of nitration under acidic conditions. Eur J Oral Sci 117: 555-562, 2009.

18. Warburg $\mathrm{OH}(\mathrm{ed})$ : The metabolism of tumours: investigations from the Kaiser Wilhelm Institute for Biology, Berlin-Dahlem. Richard R. Smith Inc., New York, pp129-169, 1931.

19. Hirayama A, Kami K, Sugimoto M, Sugawara M, Toki N Onozuka H, Kinoshita T, Saito N, Ochiai A, Tomita M, et al: Quantitative metabolome profiling of colon and stomach cancer microenvironment by capillary electrophoresis time-of-flight mass spectrometry. Cancer Res 69: 4918-4925, 2009.
20. Ackerstaff E, Glunde K and Bhujwalla ZM: Choline phospholipid metabolism: A target in cancer cells? J Cell Biochem 90: 525-533, 2003

21. Katz-Brull R, Seger D, Rivenson-Segal D, Rushkin E and Degani H: Metabolic markers of breast cancer: Enhanced choline metabolism and reduced choline-ether-phospholipid synthesis. Cancer Res 62: 1966-1970, 2002

22. Haddadin IS, McIntosh A, Meisamy S, Corum C, Styczynski Snyder AL, Powell NJ, Nelson MT, Yee D, Garwood M and Bolan PJ: Metabolite quantification and highfield MRS in breast cancer. NMR Biomed 22: 65-76, 2009.

23. Katz-Brull R, Margalit R and Degani H: Differential routing of choline in implanted breast cancer and normal organs. Magn Reson Med 46: 31-38, 2001

24. Harris RA, Zhang B, Goodwin GW, Kuntz MJ, Shimomura Y, Rougraff P, Dexter P, Zhao Y, Gibson R and Crabb DW: Regulation of the branched-chain alpha-ketoacid dehydrogenase and elucidation of a molecular basis for maple syrup urine disease. Adv Enzyme Regul 30: 245-263, 1990.

25. Mustafa A, Gupta S, Hudes GR, Egleston BL, Uzzo RG and Kruger WD: Serum amino acid levels as a biomarker for renal cell carcinoma. J Urol 186: 1206-1212, 2011.

26. Ma Y, Zhang P, Wang F, Liu W, Yang J and Qin H: An integrated proteomics and metabolomics approach for defining oncofetal biomarkers in the colorectal cancer. Ann Surg 255: 720-730, 2012.

27. Gaudet MM, Falk RT, Stevens RD, Gunter MJ, Bain JR, Pfeiffer RM, Potischman N, Lissowska J, Peplonska B, Brinton LA, et al: Analysis of serum metabolic profiles in women with endometrial cancer and controls in a population-based casecontrol study. J Clin Endocrinol Metab 97: 3216-3223, 2012.

28. Cross AJ, Moore SC, Boca S, Huang WY, Xiong X, StolzenbergSolomon R, Sinha R and Sampson JN: A prospective study of serum metabolites and colorectal cancer risk. Cancer 120: 3049-3057, 2014

29. Willems L, Jacque N, Jacquel A, Neveux N, Maciel TT, Lambert M, Schmitt A, Poulain L, Green AS, Uzunov M, et al: Inhibiting glutamine uptake represents an attractive new strategy for treating acute myeloid leukemia. Blood 122: 3521-3532, 2013.

30. Ye N, Liu C and Shi P: Metabolomics analysis of cervical cancer, cervical intraepithelial neoplasia and chronic cervicitis by $1 \mathrm{H}$ NMR spectroscopy. Eur J Gynaecol Oncol 36: 174-180, 2015.

31. Deja S, Porebska I, Kowal A, Zabek A, Barg W, Pawelczyk K, Stanimirova I, Daszykowski M, Korzeniewska A, Jankowska R, et al: Metabolomics provide new insights on lung cancer staging and discrimination from chronic obstructive pulmonary disease. J Pharm Biomed Anal 100: 369-380, 2014.

32. De Luca V, Viggiano E, Messina G, Viggiano A, Borlido C, Viggiano A and Monda M: Peripheral amino acid levels in schizophrenia and antipsychotic treatment. Psychiatry Investig 5: 203-208, 2008.

33. Liang Q, Wang C and Li B: Metabolomic analysis using liquid chromatography/mass spectrometry for gastric cancer. Appl Biochem Biotechnol 176: 2170-2184, 2015.

34. Shirai M: Transcription regulation of the urease operon in Helicobacter pyiori in response to $\mathrm{pH}$ and mechanisms of stable colonizaion in the stomach. Yamaguchi Med 50: 593-601, 2001.

35. Hilvo M, de Santiago I, Gopalacharyulu P, Schmitt WD, Budczies J, Kuhberg M, Dietel M, Aittokallio T, Markowetz F, Denkert C, et al: Accumulated metabolites of hydroxybutyric acid serve as diagnostic and prognostic biomarkers of ovarian high-grade serous carcinomas. Cancer Res 76: 796-804, 2016.

36. Zeng J, Yin P, Tan Y, Dong L, Hu C, Huang Q, Lu X, Wang H and Xu G: Metabolomics study of hepatocellular carcinoma: Discovery and validation of serum potential biomarkers by using capillary electrophoresis-mass spectrometry. J Proteome Res 13: 3420-3431, 2014.

37. OuYang D, Xu J, Huang $\mathrm{H}$ and Chen Z: Metabolomic profiling of serum from human pancreatic cancer patients using $1 \mathrm{H}$ NMR spectroscopy and principal component analysis. Appl Biochem Biotechnol 165: 148-154, 2011.

38. Hur H, Paik MJ, Xuan Y, Nguyen DT, Ham IH, Yun J, Cho YK, Lee $\mathrm{G}$ and Han SU: Quantitative measurement of organic acids in tissues from gastric cancer patients indicates increased glucose metabolism in gastric cancer. PLoS One 9: e98581, 2014. 